\title{
Performance Analysis of a Forwarding Scheme for Handoff in HAWAII
}

\author{
Chris Blondia $^{1}$, Olga Casals ${ }^{2}$, Llorenç Cerdà ${ }^{2}$, and Gert Willems ${ }^{1}$ \\ 1 University of Antwerp, Dept. Mathematics and Computer Science, \\ Universiteitsplein 1, B-2610 Antwerpen, Belgium \\ \{blondia, gewillem\}@uia.ua.ac.be \\ http://win-www.uia.ac.be/u/pats/ \\ 2 Polytechnic University of Catalunia, Computer Architecture Dept., \\ Gran Capitan, D-6, E-08071 Barcelona, Spain \\ $\{$ olga, llorenc\}@ac.upc.es \\ http://research.ac.upc.es/XARXES/CompNet/
}

\begin{abstract}
Demand for mobile network access is having a huge increase nowadays. Cellular networks are being deployed to cope with a high number of users. Several IP micro-mobility protocols have been proposed to handle routing and handoffs inside cellular networks. In this paper the HAWAII micro-mobility protocol is analyzed by means of an analytical model. A detailed description of the handoff procedure is given and illustrated by means of traces obtained from simulation. Several of the system details are taken into account in the analytical model. This allows us to investigate the influence of various system parameters (e.g. cell overlap area, beacon latency, forwarding buffer capacity, etc.) on the system performance for constant bit rate (UDP) traffic. The results are validated by means of simulation results obtained with the network simulator (ns).
\end{abstract}

\section{Introduction}

Handheld computing devices, such as palmtop computers are becoming the preferred platform for nowadays personal applications. With the evolution of these devices from having a limited communication support, typical point-to-point interfaces (PSTN modem or RS-232 cable), towards high-speed packet radio access interfaces, the demand for network access to mobile users will grow exponentially. As demands increase for wireless communication services, such as high-speed Internet access, video and high-quality image transmission, the wireless network access infrastructure will have to support a variety of applications and access speeds which should result in a service with the same level of quality as wireline users. Higher speed can be achieved in a cellular network by considering smaller cells. However, the smaller the cells are, the higher the frequency of handoffs may be. Mobile IP [1] (MIP), the current support of mobility in IP networks, delivers packets to a temporary address assigned to the mobile host at its current point of attachment. This temporary address is communicated to a possibly distant Home Agent. This approach applied to an environment with

E. Gregori et al. (Eds.): NETWORKING 2002, LNCS 2345, pp. 503-514 2002.

(C) Springer-Verlag Berlin Heidelberg 2002 
frequent handoffs may lead to high associated signalling load and unacceptable disturbance to ongoing sessions in terms of handoff latency and packet losses. Therefore, a hierarchical mobility management approach has been proposed (see e.g. [2]), where MIP supports wide area mobility (e.g. mobility between different operators) while local mobility is handled by more optimized micro-mobility protocols. These protocols should incorporate a number of important design features related to location management, routing and handoff schemes. They should fulfill requirements such as simplicity to implement, scalability with respect to the induced signalling, efficiency and performance with respect to packet loss and introduced delay. Prominent solutions for micro-mobility support are HAWAII (5]) and Cellular IP ([4]).

In this paper, the performance of a handoff scheme based on packet forwarding used in HAWAII is evaluated by means of a simulation study and analytical modeling. The models that are presented allow to compute characteristic performance measures related to packet loss due to the expiration of the playout time and the delay experienced by packets involved in the handoff, together with the influence of various system characteristics on these performance measures. In [5], the expected number of dropped or lost packets as a function of the playout time has already been investigated. The aim of this study was to compare different path setup schemes with MIP and MIP with Route Optimization. Contrary to our paper, [5] does not investigate the influence of system details such as cell overlap area, beacon signal latency, time out and capacity of the forwarding buffer, on the system performance.

The remainder of this paper is structured as follows. In section 2, the HAWAII protocol and the forwarding scheme for handoff is explained. A detailed description of the protocol as implemented in the ns simulator is made in Section 3. In Section 4 a simple analytical model to assess the packet loss and the experienced packet delay due to handoff is presented. Section 5 is devoted to numerical results, showing the influence of the different system parameters on the performance using the analytical model. A model validation is made based on simulation results. Finally section 6 concludes the paper.

\section{A Forwarding Scheme for Handoff in HAWAII}

HAWAII is a domain-based approach for supporting mobility ([5] and [3]). The gateway into each domain is called the domain root router. The Mobile Host $(\mathrm{MH})$ keeps it network address unchanged while moving within a domain. The Corresponding Hosts $(\mathrm{CH})$ and the Home Agent (HA) do not need to be aware of the host's position within the domain. To reach the MH, HAWAII uses specialized path setup schemes that update forwarding entries in specific routers. When a router receives a packet for an unknown $\mathrm{MH}$, it uses a preconfigured default interface pointing towards the domain root router. The packet will be forwarded in that direction till it arrives at a router knowing a route to the MH. There are two classes of path setup schemes for updating routing information: one for networks with MHs that can only maintain connection to one base station (e.g. 


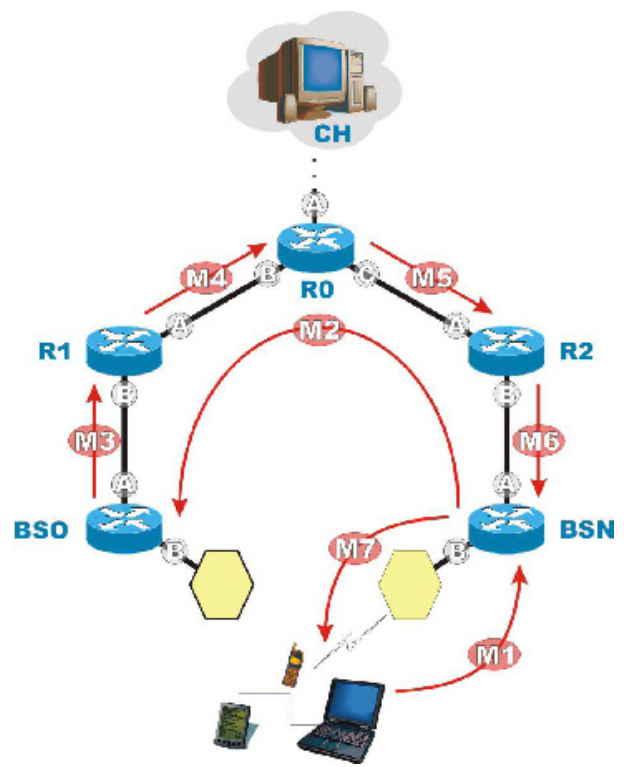

Fig. 1. Messages in MSF.

TDMA networks) and the other one for networks with MHs that can be connected to two or more base stations simultaneously like in CDMA networks for example. The first class includes two forwarding path setup schemes: the Multiple Stream Forwarding (MSF) and an alternative, the Single Stream Forwarding (SSF) scheme. These schemes forward packets from the Old Base Station (BSO) to the New Base Station (BSN) before being diverted at the crossover router (i.e. a router where the path from $\mathrm{CH}$ to BSO and the path from BSO to BSN cross). The second class includes two non-forwarding schemes: the Unicast NonForwarding (UNF) scheme and the Multicast Non-Forwarding (MNF) scheme. In these last schemes the BSO does not forward any packets to BSN. In this paper we limit our analysis to the MSF scheme although a similar analytical model can be applied to evaluate other schemes 6]. For the description of the MSF handoff protocol and its performance evaluation, we will use the following reference network (see Fig. 1). Let a MH move from the cell controlled by BSO to the cell controlled by BSN. The corresponding cells have a non-empty overlap area. Packets originating from the Corresponding Host $(\mathrm{CH})$ reach BSO (resp. BSN) via the crossover router $\mathrm{R} 0$ and the intermediate router $\mathrm{R} 1$ (resp. R2). When the handoff is initiated, the MH closes the connection with BSO, establishes a connection to BSN and sends a MIP registration message (M1) to BSN, which in turn sends a path setup update message M2 to the BSO. All remaining packets arriving at BSO are stored in a buffer and forwarded to BSN when M2 arrives. Furthermore, when M2 arrives, BSO sends the path setup message (M3) to R1, who adds a forwarding entry to its routing table indicating that packets for the $\mathrm{MH}$ should leave the R1 via interface A. R1 sends the path setup message (M4) 
to R0, who adds a forwarding entry indicating that packets for the MH should leave the R0 via interface $\mathrm{C}$. From this instant on, all packets arriving at router R0 are sent directly to BSN. The path setup message continues (M5 and M6) triggering similar actions until it reaches BSN. Remark that MSF can create transient routing loops (for example after BSO has changed its entry to forward packets but before R1 processes M3).

\section{Detailed Description of the Handoff Procedure in the MSF Scheme}

In this section we give a detailed description of a possible implementation of the MSF handoff procedure that corresponds to the one programmed in the network simulator (ns) used for the simulation analysis. We show the system parameters that may have a major impact on the handoff performance. These parameters will be considered in the performance evaluation.

All BS generate beacon signals at regular instants. The $\mathrm{MH}$ connects to the BS with the strongest beacon signal power. This implies that if a MH, connected to BSO, moves towards BSN it will initiate a handoff and connect to this Base Station after receiving the first beacon signal which was generated by BSN after the MH passed the middle of the overlap area of the cells controlled by BSO and BSN. Remark that as long as the $\mathrm{MH}$ is in the overlap area and the beacon of $\mathrm{BSN}$ is not generated, the MH stays connected to BSO and thus can still receive packets from BSO. Remember from Section 2 that BSN sends a path setup update message (M2) to BSO. BSO continues to send the packets to the $\mathrm{MH}$, until M2 arrives at BSO. These packets will reach the $\mathrm{MH}$ only if it is inside the coverage of BSO. BSO uses a circular buffer of size FB packets to store the packets to be forwarded to BSN. We refer to this buffer as the forwarding buffer. All packets addressed to the MH are stored in this buffer (even after being transmitted to the $\mathrm{MH}$ ). This allows packets, sent to the $\mathrm{MH}$ but lost because the $\mathrm{MH}$ moved out of coverage, to reach the $\mathrm{MH}$ when forwarded to BSN. Furthermore, the forwarding buffer is provided with a time out mechanism that ensures that a packet is held by the buffer only for a limited time period. When the path setup update message (M2) arrives at BSO, all packets outstanding in the buffer for which the time out is not expired are forwarded to BSN. Note that some of these packets may already have been successfully delivered to the MH.

Figure 2 illustrates the handoff procedure. The trace depicted in the figure has been obtained using the values of one of the ns simulations described later in this paper. The $\mathrm{x}$-axis corresponds to the instants upon which new packets arrive at the $\mathrm{MH}$, and the $\mathrm{y}$-axis to the end-to-end delay of each packet. The figure shows the following time instants: the moment that the $\mathrm{MH}$ reaches the middle of the overlapping area of the two cells (intercell crossing), the instant when the MH crosses the border of the cell controled by BSO (end of coverage), the time instant at which the beacon is sent by BSN, and the moment the forwarding message M2 sent by BSN reaches BSO. For each arrived packet, the figure also indicates if it was sent by BSO, BSN or forwarded. The forwarded packets are those that are 


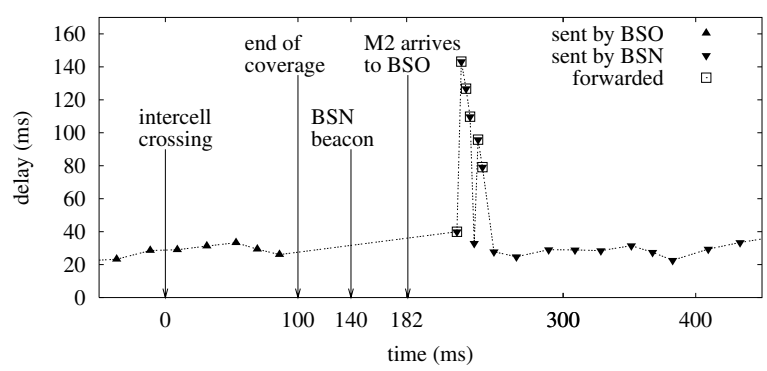

Fig. 2. Handoff procedure in the MSF.

turned around by some router (BSO or any intermediate router between BSO and the crossover router R0) because of the handoff. In this simulation a FB size of 10 packets is used with a time out of $100 \mathrm{~ms}$. When the M2 message arrives at BSO, 6 packets are forwarded from BSO: the first one had already arrived at the $\mathrm{MH}$ (and thus is a duplicated packet not shown as forwarded in the figure). The other five are received by BSO between the end of coverage instant and the M2 message arrival instant. These packets are able to reach the MH after being forwarded to BSN and hence have a higher delay. Finally, note that the first forwarded packet arrives at BSN with a lower delay than the others. This is because this packet was turned around by a router located before BSO (this packet arrived at this router after the router was being updated with the M3 message sent by BSO).

\section{An Analytical Model of the Forwarding Schemes}

We introduce the following notations. Let $R x(X)$ be the random variable denoting the time needed for a packet to be processed by router Rx and leaving via interface X. In other words, it denotes the time between the arrival of a packet at the router and its departure through the output interface X. (Same notation applies to "routers" BSO and BSN). Let $(R x, R y)$ denote the propagation time on the link between router $\mathrm{Rx}$ and router Ry. Furthermore, we denote by $\left(R x \rightarrow R z^{\sigma}\right)$ the time needed for a packet (or message) to travel from $\mathrm{Rx}$ to $\mathrm{Rz}$. The superscript ${ }^{\sigma}$ indicates that the processing of the specific router is included. Assume that the time (in ms) the MH needs to cross the overlap area is given by $o$ ms. Finally, let $\tau_{b}$ denote the beacon latency, i.e. the difference between the time instant the beacon signal is generated and the instant the $\mathrm{MH}$ reaches the end of BSO's coverage area.

We now present a mathematical model for the MSF scheme. The following assumption is essential for computational tractability reasons. All routers involved in the path setup scheme are modeled as simple $M / M / 1$ queues. The exponential service time of a packet includes both the processing time and the transmission time. Denote the load of a router by $\rho$ and the exponential service rate by $\mu$. Hence, the random variable $R x(X)$, being the response time in an 
$M / M / 1$ queue, is exponentially distributed with rate $\mu(1-\rho)$. With this assumption, $\left(R x \rightarrow R z^{\sigma}\right)$ is the sum of exponential variables (e.g. $\left.R x(X)\right)$ and fixed propagation delays (e.g. $(R x, R y))$.

The packets involved in a handoff can be divided in classes according to the path they follow. The timing of these classes is given from the point of view of the arrival of a packet at router R0. Denote $t_{m}$ as the instant the MH passes the middle of the overlap area, or the instant the $\mathrm{MH}$ crosses the border between 2 cells when there is no overlap. We consider all those packets that arrive at BSO after this instant as involved in the handoff and divide them in 4 classes.

- Class 1: These packets are processed by the BSO after $t_{m}$ and before the new forwarding entry is added in the tables of the BSO. They arrive at R0 after

$$
t_{0}=t_{m}-\left(R 0^{\sigma} \rightarrow B S O^{\sigma}\right)
$$

but before

$$
t_{1}=t_{m}+\frac{o}{2}+\tau_{b}+\left(M H \rightarrow R 0^{\sigma}\right)
$$

Class 1 packets are either directly sent to the $\mathrm{MH}$, or they will eventually be forwarded to the BSN from the FB, unless they are removed from this buffer due to time out expiration or buffer overflow.

- Class 2: These packets arrive at R1 before M3 causes the adding of the new forwarding entry and they arrive at the BSO after its forwarding table has been updated by M2. Those packets are therefore directly forwarded from BSO to BSN without being put in the forwarding buffer. At time instant

$$
t_{1}^{\prime}=t_{1}+\left(R 0 \rightarrow B S O \rightarrow R 1^{\sigma}\right)
$$

M3 is processed and router R1 changes its forwarding entries for packets with destination the MH. Hence, packets arriving at R0 in the interval $\left[t_{1}, t_{2}\right]$ with

$$
t_{2}=t_{1}^{\prime}-\left(R 0^{\sigma} \rightarrow R 1\right)
$$

belong to class 2 .

- Class 3: These packets arrive at R0 before M4 causes the adding of the new forwarding entry at R0, and they arrive at R1 after $t_{1}^{\prime}$. At time instant

$$
t_{3}=t_{2}+\left(R 0^{\sigma} \rightarrow R 1\right)+\left(R 1 \rightarrow R 0^{\sigma}\right)
$$

router $\mathrm{R} 0$ changes its forwarding entries for packets with destination the $\mathrm{MH}$. Therefore class 3 packets are those arriving at R0 in $\left[t_{2}, t_{3}\right]$.

- Class 4: These packets arrive at R0 after time instant $t_{3}$ and are directly diverted towards BSN. They experience no extra delay due to the handoff.

Consider a constant bit rate stream of packets originating from the $\mathrm{CH}$, arriving at router $\mathrm{R} 0$ with a constant interarrival time of $T$ ms. We let the arrival instant $u$ of the first packet be uniformly distributed over $\left[t_{0}, t_{0}+T\right]$ and set $t_{0}=0$. Then it is possible to compute the probability distribution of the end-to-end delay for each packet traveling from the $\mathrm{CH}$ to the $\mathrm{MH}$. Consider the $k$-th packet, arriving at R0 at time instant $(k-1) T+u$, and denote its end-to-end delay by $(\mathrm{e}-\mathrm{e})_{k}$. The probability that $(\mathrm{e}-\mathrm{e})_{k}$ is larger than $t$, depends basically on the class it belongs to and is given by the following expression.

$$
\begin{aligned}
& \mathrm{P}\left[(\mathrm{e}-\mathrm{e})_{k}>t\right]= \\
& \mathrm{P}\left[(k-1) T+u<t_{1}\right] \mathrm{P}\left[(\mathrm{e}-\mathrm{e})_{k}>t \mid(k-1) T+u<t_{1}\right] \\
& +\mathrm{P}\left[t_{1}<(k-1) T+u<t_{2}\right] \mathrm{P}\left[(\mathrm{e}-\mathrm{e})_{k}>t \mid t_{1}<(k-1) T+u<t_{2}\right] \\
& +\mathrm{P}\left[t_{2}<(k-1) T+u<t_{3}\right] \mathrm{P}\left[(\mathrm{e}-\mathrm{e})_{k}>t \mid t_{2}<(k-1) T+u<t_{3}\right] \\
& +\mathrm{P}\left[t_{3}<(k-1) T+u\right] \mathrm{P}\left[(\mathrm{e}-\mathrm{e})_{k}>t \mid t_{3}<(k-1) T+u\right]
\end{aligned}
$$


For class 2, 3 and 4 respectively, we obtain

$$
\begin{aligned}
& \mathrm{P}\left[(\mathrm{e}-\mathrm{e})_{k}>t \mid t_{1}<(k-1) T+u<t_{2}\right]=\mathrm{P}\left[\left(C H \rightarrow B S O \rightarrow R 0 \rightarrow B S N^{\sigma}\right)>t\right] \\
& \mathrm{P}\left[(\mathrm{e}-\mathrm{e})_{k}>t \mid t_{2}<(k-1) T+u<t_{3}\right]=\mathrm{P}\left[\left(C H \rightarrow R 1 \rightarrow R 0 \rightarrow B S N^{\sigma}\right)>t\right] \\
& \mathrm{P}\left[(\mathrm{e}-\mathrm{e})_{k}>t \mid t_{3}<(k-1) T+u\right]=\mathrm{P}\left[\left(C H \rightarrow R 0 \rightarrow B S N^{\sigma}\right)>t\right] .
\end{aligned}
$$

The probability for a class 1 packet depends on $k$ and is quite complicated as this involves the length of the overlap area, the instant the beacon is sent, the time out in the forwarding buffer and the size of this buffer. We distinguish three different cases for a class 1 packet:

- case 1: the $k$-th packet is directly sent from BSO to $\mathrm{MH}$.

- case 2: the $k$-th packet is forwarded to MH via BSN.

- case 3: the $k$-th packet is lost at BSO (due to time out or buffer overflow).

For case 1 we obtain

$$
(\mathrm{e}-\mathrm{e})_{k}=\left(C H \rightarrow R 0 \rightarrow B S O^{\sigma}\right)
$$

for case 2

$$
(\mathrm{e}-\mathrm{e})_{k}=\left(C H \rightarrow R 0 \rightarrow B S O^{\sigma}\right)+\left(t_{1}-(k-1) T-u\right)+\left(B S O^{\sigma} \rightarrow R 0 \rightarrow B S N^{\sigma}\right)
$$
and for case 3 the end-to-end delay can be considered to be infinite. In case 2 , we consider a possible loop between $\mathrm{BSO}$ and $\mathrm{R} 1$ for the first few packets that are forwarded from the FB, and we account for possible extra delay due to the burst of packets that is created when the FB is emptied. The details are omitted in this paper. We also need to determine the probability that the $k$-th packet finds itself in case 1, 2 or 3 respectively. Again most details are omitted and case 3 is considered as an example. Clearly,

$\mathrm{P}[k$-th packet is in case 3$]=\mathrm{P}\left[\left(k\right.\right.$-th packet is processed by BSO after $\left.t_{d}\right) \mathrm{AND}$ ( $k$-th packet is timed out OR pushed out of the $\mathrm{FB})]$,

where $t_{d}$ is the instant of disconnection of the $\mathrm{MH}$ from the BSO, directly depending on the values of $o$ and $\tau_{b}$. This probability yields a rather complex expression in which the probability that a packet is pushed out of the circular buffer FB before it could be forwarded is required:

$\mathrm{P}[k$-th packet is pushed out $]=\mathrm{P}\left[(k+\right.$ buffersize- 1$\left.) T+u<t_{1}\right]$

which is the probability that the ( $k+$ buffersize)-th packet needs to be forwarded (and therefore pushes out the $k$-th packet). The probablity that a packet is timed out is also required:

$\mathrm{P}[k$-th packet is timed out $]=\mathrm{P}\left[t_{p_{k}}<t_{1}+\left(R 0 \rightarrow B S O^{\sigma}\right)-T O\right]$

where $t_{p_{k}}$ denotes the instant of the end of the processing of the $k$-th packet at $\mathrm{BSO}$ and $T O$ is the time out value. Remark that $t_{1}+\left(R 0 \rightarrow B S O^{\sigma}\right)$ equals the instant that the FB is emptied and the buffered packets are forwarded.

It is clear that, due to the $M / M / 1$ assumption, all probabilities that occur in the above formulas can be computed through some standard conditional probability techniques. Similarly, the model allows us to compute several performance measures such as the expected number of packets arriving late at a playout buffer in the $\mathrm{MH}$, due to the extra delay introduced by the forwarding scheme. 


\section{Performance Evaluation of the MSF Scheme}

In this section we consider three cases in order of increasing complexity. In the first case (Section 5.1), cells do not overlap $(o=0 \mathrm{~ms})$ and the beacon signal is received by the $\mathrm{MH}$ at the moment that it crosses the border between the cell controlled by BSO and the cell controlled by BSN. The time out (TO) and the capacity of the forwarding buffer in BSO $(F B)$ are supposed to be large enough so that they do not cause packet loss. In the second case (Section 5.2) the

(A)

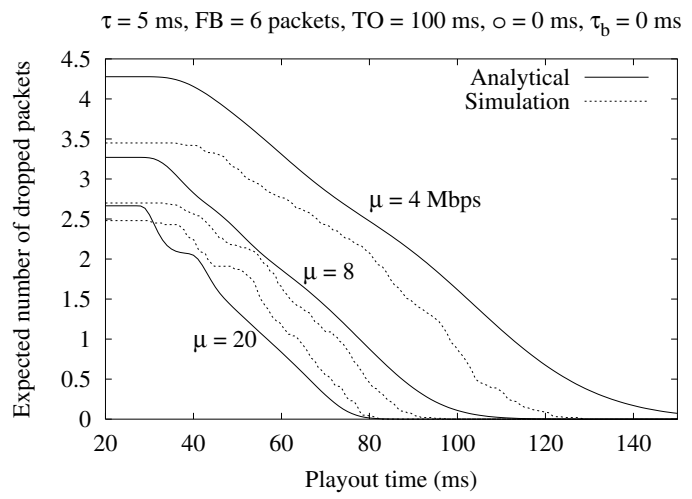

$\mu=4 \mathrm{Mbps}, \mathrm{FB}=15$ packets, $\mathrm{TO}=200 \mathrm{~ms}, \circ=0 \mathrm{~ms}, \tau_{\mathrm{b}}=0 \mathrm{~ms}$

(B)

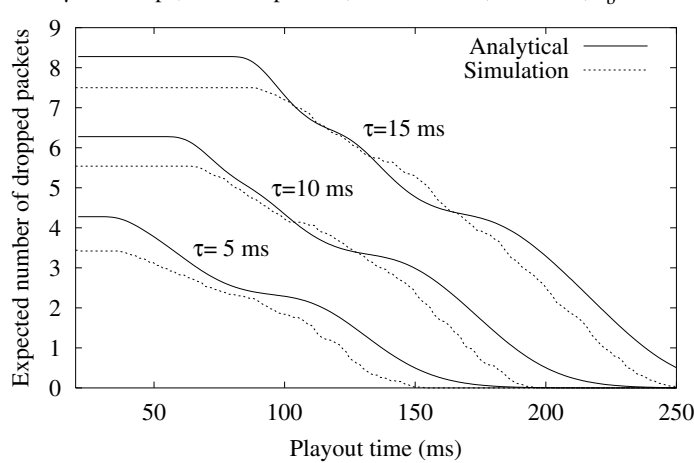

Fig. 3. Expected number of dropped packets vs. playout time for variable transmission rate $\mu(\mathrm{A})$ and for variable link delays (B).

cells overlap $(o>0 \mathrm{~ms})$. The first beacon sent after the $\mathrm{MH}$ crosses the middle of the overlap area (determining the handoff instant) may occur while the $\mathrm{MH}$ is in the overlap area or after the MH has left the overlap area. Again in this case, no packets will be lost due to time out and/or forwarding buffer overflow. In the third case (Section 5.3) we have the same characteristics as the second one, except that the time out value and the forwarding buffer capacity are chosen so that packets may be lost in the forwarding buffer. In all three cases we consider 


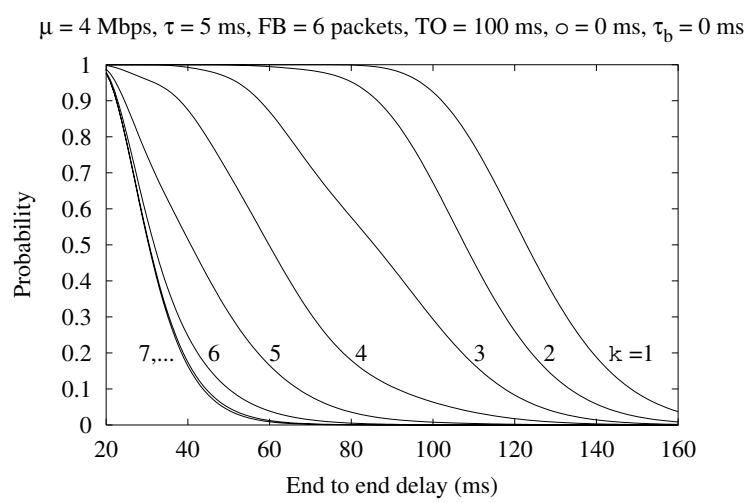

Fig. 4. Delay Distribution of $k$-th Packet.

the system shown in Fig. 1]where: (i) the fixed propagation delay between routers $(R x, R y)$ is the same for all routers (also between $\mathrm{CH}$ and $\mathrm{R} 0$ ) and we will refer to it as $\tau$, (ii) the correspondent node $(\mathrm{CH})$ transmits 500 byte packets every 20 ms to the MH, (iii) the background traffic is modelled as Poisson sources such that each router has a load $\rho$ of 0.8 .

\subsection{Delay Evaluation}

The playout time is the maximum allowed end-to-end delay: if a packet's endto-end delay exceeds this playout time, it will be dropped. In Fig. 3 the expected number of forwarded packets that are dropped due to expiration of playout time is shown as a function of the playout time, for different values of the transmission rate $\mu$ in the routers in Fig. 3 $\mathrm{A}$ and for different values of distance $\tau$ between neighbouring routers in Fig. 3.B .

The analytical results are compared against simulation results. The difference between simulation and analytical results is due to the $M / M / 1$ approximation in the analytical model resulting in exponential packet service times, while in the simulation packets have constant length.

Fig. 4 depicts the distribution of the end-to-end delay of the $k$-th packet involved in the handoff when there is no overlap between the cells. As can be expected, the delay decreases with increasing sequence number of the packet. Starting from packet 7 , the curves converge since the probability to experience some extra delay due to forwarding decreases rapidly.

\subsection{Influence of the Beacon Latency}

Fig. [5] shows the important influence the beacon latency has on the system performance. The expected number of forwarded packets dropped due to expiration of playout time is shown as a function of the playout time, for different values of the time between the instant the $\mathrm{MH}$ crosses the coverage area of BSO and the 


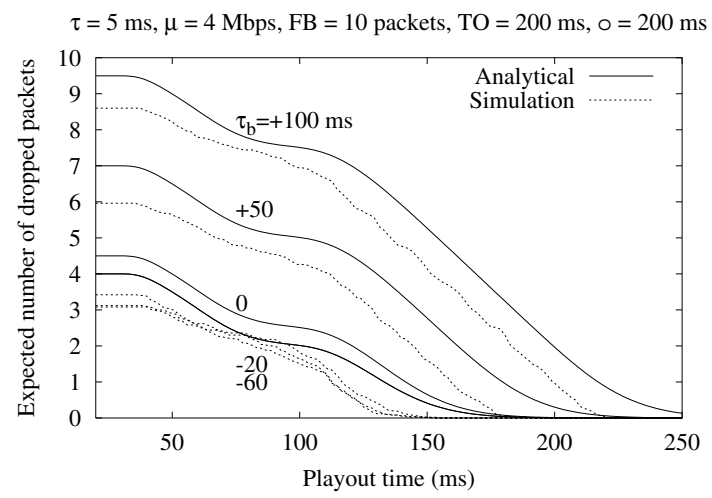

Fig. 5. Expected number of dropped packets vs. playout time for variable beacon latency $\tau_{b}$.

instant that the first beacon originating from the BSN is received. A positive value of $\tau_{b}$ means that the beacon arrives after the end of the coverage area of $\mathrm{BSO}$, while a negative value means that the beacon arrives before the end of the coverage area of BSO. Again the analytical results are validated with the ns simulation. When the beacon arrives after the end of the coverage area of BSO a higher number of packets will have to be stored in the forwarding buffer of the BSO which have to be forwarded to BSN once the path setup message triggered by the beacon arrival is received in BSO. This is shown in Fig. 2 for a beacon latency of $40 \mathrm{~ms}$. This forwarding increases the end-to-end delay and therefore a higher number of packets will be dropped for a given playout time. Fig. [6 shows the delay distribution of the packets involved in the handoff procedure (i.e. the packets directly sent to the $\mathrm{MH}$ or forwarded after the instant the $\mathrm{MH}$ crosses the middle of the overlap area). From this figure, it is clear that the first packet

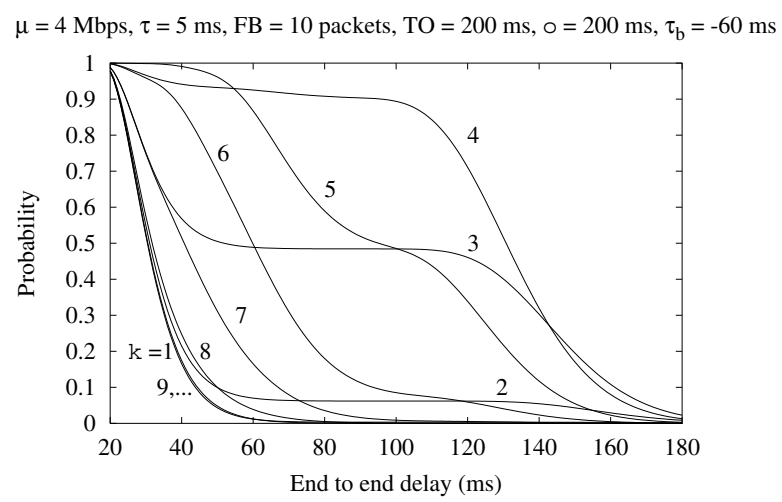

Fig. 6. Delay Distribution of $k$-th packet. 
has a high probability to be sent directly to the MH without being forwarded via the BSN. Starting from packet 2, the probability of being forwarded increases. While these packets have a high probability to belong to class 1 , starting from packet 7 , the probability to belong to class 2 or 3 increases. Packet 9 and the following packets have a high probability to be sent directly to the MH via R2 and BSN and therefore, their delay distribution is close to the one of packet 1 .

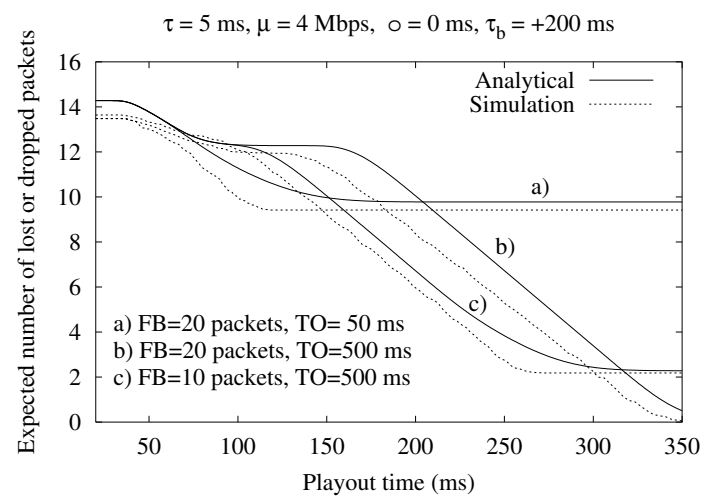

Fig. 7. Expected number of lost or dropped packets vs. playout time for variable $F B$ and $T O$.

\subsection{Influence of the BSO Time Out and Forwarding Buffer Capacity}

Forwarded packets may be dropped due to the expiration of the playout time or they may be lost when the time out in the FB expires or when they are pushed out of the circular FB when it is full. The expected number of packets dropped or lost is shown in Fig. 7 as a function of the playout time, for different values of the time out and different values of the capacity of the FB buffer. There is no overlap here and the beacon latency is $\tau_{b}=200 \mathrm{~ms}$.

In a) with $T O=50 \mathrm{~ms}$ and $F B=20$ some packets (+/- 10 packets) stored in the forwarding buffer will time out before the path setup message M2 reaches $\mathrm{BSO}$ and thus they will be lost. In b) with $T O=500 \mathrm{~ms}$ and $F B=20$ no packets are lost. In c) with $T O=500 \mathrm{~ms}$ and $F B=10$ only a few packets (+/2 packets) will find the forwarding buffer full with packets to be forwarded when arriving at BSO and thus will push out the packets at the head of the queue which will be lost.

\section{Conclusions}

In this paper, the MSF-HAWAII handoff protocol is analyzed by means of an analytical model. Its performance for constant bit rate real-time (UDP) traffic is 
characterized by two measures: the expected number of forwarded packets that are dropped due to the expiration of the playout time together with the expected number of packets lost in the forwarding buffer and secondly, the individual endto-end delay distributions of the packets that are involved in the handoff. The model includes a number of system implementation characteristics that have a major impact on the system performance: size of the overlap area between neighboring cells, frequency of beacon signal generation, size of forwarding buffer and time out value used in the forwarding buffer. The numerical results obtained with the analytical model have been validated with the ns simulator, showing the accuracy of the model.

Application of the model to a simple reference network shows that longer forwarding routes (due to longer distances between routers, slower routers or low capacity of transmission links) lead to a higher number of expected packets lost due to the expiration of the playout time and longer delays experienced by individual packets. Furthermore, it is shown that the expected number of lost packets may drastically increase when the beacon signal reaches the MH after it left the area covered by the old base station. The numerical examples also show that engineering accurately the forwarding buffer (both its time out value and its capacity) is an important, but difficult task, as unappropriate values of time out or buffer capacity may lead to a major performance degradation due to the loss of several packets.

Acknowledgments. This work was supported in part by the EU, under project IST-11591, MOEBIUS; The 2nd and 3th author were supported by the Ministry of Education of Spain, grant TIC-1998-115-C02-01

\section{References}

1. C. Perkins, ed., "IP Mobility Support", IETF RFC 2002, October 1996.

2. R. Caceres and V. Padmanabhan, "Fast and scalable handoffs for wireless networks", in Proc. ACM MOBICOM '96, pp. 56-66, 1996

3. R. Ramjee, T. La-Porta, S. Thuel, K. Varadhan, L. Salgarelli, "IP micro-mobility support using HAWAII", Internet draft, July 2000.

4. A. Valko, "Cellular IP - a new approach of Internet host mobility", ACM Computer Communication Reviews, January 1999

5. Ramjee, R., La Porta, T., Thuel, S., Varadhan, K., and Wang, S., HAWAII: a domain based approach for supporting mobility in wide-area wireless networks, Proceedings of International Conference on Network Protocols, ICNP'99.

6. Blondia, C., Casals, O., De Cleyn. P., and Willems, G., "Performance analysis of IP micro-mobility handoff protocols", Proceedings 7th Int. Workshop on Protocols for High Speed Networks, PfHSN 2002

7. A. Campbell, J. Gomez, C. Y Wan, S. Kim, Z. Turanyi, A. Valko, "Cellular IP", IETF draft (draft-ietf-mobileip-cellularip-00.txt), January 2000.

8. A. Campbell, J. Gomez, S. Kim, A. Valko, C.-Y. Wan and Z. Turanyi, "Design, implementation and evaluation of Cellular IP", IEEE Personal Communications, August 2000, pp.42-49 